\title{
Uso da penicilina para tratar uma pneumonia lobar em paciente pediátrico com deficiência de glicose-6-fosfato desidrogenase: relato de caso e revisão da literatura
}

\author{
Penicillin to treat lobar pneumonia in pediatric patient with glucose-6-phosphate \\ dehydrogenase deficiency: case report and review of the literature
}

\section{Carlos Felipe Nogueira ${ }^{1}$, Cláudio Daniel Cerdeira ${ }^{2}$, Anna Paula Mendanha da Silva Aureliano ${ }^{1}$,

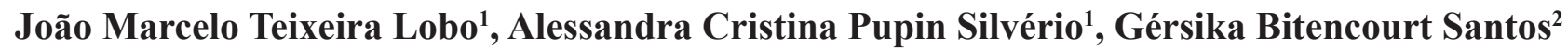

Nogueira CF, Cerdeira CD, Aureliano APMS, Lobo JMT, Silvério ACP, Santos GB. Uso da penicilina para tratar uma pneumonia lobar em paciente pediátrico com deficiência de glicose-6-fosfato desidrogenase: relato de caso e revisão da literatura / Penicillin to treat lobar pneumonia in pediatric patient with glucose-6-phosphate dehydrogenase deficiency: case report and review of the literature. Rev Med (São Paulo). 2017;96(3):177-82.

RESUMO: Objetivo: Neste relato de caso, nós abordamos o uso da penicilina em paciente pediátrico do sexo feminino com deficiência de glicose-6-fosfato desidrogenase (G6PD) para tratar uma pneumonia lobar aguda. Descrição do caso: Durante os 10 dias de tratamento com penicilina G cristalina (200 mg/kg/dia), o monitoramento com hemograma evidenciou a restauração aos níveis normais do hematócrito e hemoglobina. O acompanhamento da paciente mostrou completa resolução da infecção pulmonar e não ocorrência de anemia hemolítica aguda (crise hemolítica). Comentários: A restrição quanto ao uso de alguns medicamentos em pacientes que apresentam deficiência de G6PD é um desafio para a instituição do tratamento adequado na prática clínica. Portanto, é aqui relatado o uso da penicilina por parte de uma paciente com deficiência de G6PD.

Descritores: Deficiência de glucosefosfato-desidrogenase; Penicilinas; Anemia hemolítica; Pneumonia.
SUMMARY: Objective: In this case report, we addressed the use of penicillin in a 6-year-old girl with glucose-6-phosphate dehydrogenase (G6PD) deficiency to treat an acute lobar pneumonia. Case description: During a 10-day sequential therapy with Crystalline Penicillin G (200 mg/kg/day), blood count evidenced restoration to normal levels of hematocrit and hemoglobin. The patient showed complete resolution of pulmonary infection at the follow-up and non-occurrence of acute hemolytic anemia. Comments: The applied restrictions as for the use of some drugs by patients with G6PD deficiency is a challenge for the institution of a suitable treatment at the clinical practice. Therefore, here, it is reported the safe use of penicillin by a patient with G6PD deficiency.

Keywords: Glucosephosphate-dehydrogenase deficiency; Penicillins; Anemia, hemolytic; Pneumonia.

1.Faculdade de Medicina, Universidade José do Rosário Vellano (UNIFENAS), Alfenas, MG, Brasil. E-mails: carlos.felipe.16@hotmail. com, annapaulamendanha@hotmail.com,jmarcelo111@hotmail.com, alessandra.silverio@unifenas.br.

2.Departamento de Bioquímica (DBq), Instituto de Ciências Biomédicas, Universidade Federal de Alfenas (UNIFAL-MG), Alfenas, MG, Brasil. Emails: daniel.cerdeira.84@gmail.com, gersika.santos@unifenas.br.

Endereço para correspondência: Cláudio Daniel Cerdeira. DBq, Instituto de Ciências Biomédicas, UNIFAL-MG. Rua Gabriel Monteiro da Silva, 700, Prédio E, Sala 207 C. CEP: 37130-000 - Alfenas, MG, Brasil. E-mail: daniel.cerdeira.84@gmail.com. 


\section{INTRODUÇÃO}

deficiência de glicose-6-fosfato desidrogenase

-(G6PD) é uma doença hereditária recessiva, ligada ao cromossomo X. Foi descrita pela primeira vez em 1956, ao ser observado em soldados à ocorrência de anemia hemolítica induzida pelo antimalárico primaquina. Atualmente, a deficiência de G6PD afeta mais de 400 milhões de pessoas em todo o mundo e cerca de $7 \%$ da população mundial é portadora do gene G6PD mutado. A elevada prevalência desta enzimopatia tem sido observada principalmente em regiões tropicais e subtropicais ${ }^{1-4}$.

A G6PD é uma enzima citosólica catalisadora da primeira reação na via das pentoses-fosfato. Esta reação gera elétrons que são transferidos para a nicotinamida adenina dinucleótido fosfato $\left(\mathrm{NADP}^{+}\right)$, formando NADPH (forma reduzida). Nas hemácias, a via das pentoses-fosfato é a única fonte de NADPH, sendo que este composto é utilizado pela enzima glutationa-redutase (GSH-Rd) para a conversão da glutationa oxidada (GSSG) em glutationa reduzida (GSH). Adequados níveis de GSH evita o estresse oxidativo nas hemácias, protegendo a célula do ataque de oxidantes em conta do seu papel no transporte de oxigênio e desde que o estresse oxidativo pode gerar um quadro de hemólise, o que pode levar a abortos espontâneos, hiperbilirrubinemia (Icterícia) e, em casos mais graves, "kernicterus" neonatal ${ }^{3,5-9}$.

Em indivíduos com deficiência de G6PD, o estresse oxidativo nas hemácias pode ser desencadeado pelo uso de alguns medicamentos, por infecções, por ingestão de certos alimentos, ou por fatores ambientais. A abordagem mais efetiva para prevenir a crise hemolítica em pacientes com deficiência de G6PD é a gestão de situações clínicas nas quais existe a necessidade de uma terapia medicamentosa, levando-se em conta a segurança do fármaco quanto a uma possível indução de estresse oxidativo. Portanto, alguns fármacos têm sido classificados como seguros ou contraindicados para estes pacientes ${ }^{2,10-13}$.

Apesar dos esforços, a segurança quanto ao uso de alguns medicamentos por indivíduos com deficiência de G6PD permanecem ainda pouco entendidos, controversos, ou pobremente relatados. Neste relato de caso, descrevemos o uso da penicilina para tratar uma pneumonia lobar em uma paciente pediátrica com deficiência de G6PD, sem a posterior ocorrência de anemia hemolítica associada a este tratamento.

\section{RELATO DE CASO}

Este relato de caso foi conduzido em acordância com a declaração de Helsinki (1964) e com a Resolução CNS n ${ }^{\circ}$ 196/96 do Ministério da Saúde do Brasil e foi aprovado pelo Comitê de Ética local da Universidade José do Rosário Vellano (CAAE 48474715.2.0000.5143). Um Termo de Consentimento Livre e Esclarecido (TCLE) foi préviamente assinado.

MMO, 6 anos de idade, sexo feminino, $18 \mathrm{~kg}$, branca. A paciente foi diagnosticada com deficiência de G6PD no primeiro ano de vida (Tabela 1), apresentando infecções recorrentes, episódios de anemia hemolítica e icterícia neonatal prolongada e requerido hospitalização duas vezes. Na triagem neonatal, o teste do pezinho mostrou ausências de Fenilcetonúria (Phe normal), de anemia falciforme (Hb: FA), de fibrose cística (IRT normal) e de toxoplasmose e hipotireoidismo congênitas. História neonatal: sem intercorrências. Paciente com aleitamento materno exclusivo até os 05 meses de idade e calendário de vacinação de acordo com o recomendado pelo Ministério da Saúde do Brasil. A paciente não frequentou creche ou escola até o momento. Criança nascida de pais não consanguíneos. Irmão saudável, sem manifestar traços de deficiência de G6PD.

Tabela 1. Variantes de atividade da enzima glicose-6-fosfato desidrogenase (G6PD) descritas por classes de deficiência e níveis de atividade enzimática

\begin{tabular}{|c|c|c|c|c|c|}
\hline $\begin{array}{l}\text { Atividade residual } \\
\text { enzimática }\end{array}$ & Classe & Sintomas clínicos & $\begin{array}{l}\text { Nível de deficiência/ } \\
\text { Atividade enzimática }\end{array}$ & Epidemiologia & $\begin{array}{l}\text { Tipo de mutação mais } \\
\text { frequente }\end{array}$ \\
\hline $\begin{array}{l}<1 \% \text { ou não } \\
\text { detectável }\end{array}$ & I & $\begin{array}{l}\text { Anemia hemolítica não } \\
\text { esferocítica crônica }\end{array}$ & Severa/deficiente & $\begin{array}{l}\text { Condição rara, sem } \\
\text { precisa distribuição }\end{array}$ & $\begin{array}{l}\text { G6PD-Buenos Aires, } \\
\text { G6PD-Durham }\end{array}$ \\
\hline$<10 \%$ do normal & II & $\begin{array}{l}\text { Anemia hemolítica } \\
\text { aguda* }\end{array}$ & Severa/deficiente & $\begin{array}{l}\text { Condição frequente, } \\
\text { distribuída ao longo do } \\
\text { mundo }\end{array}$ & $\begin{array}{l}\text { G6PD-Mediterranean, } \\
\text { G6PD-Cassano, } \\
\text { G6PD-Santamaria }\end{array}$ \\
\hline $10-60 \%$ do normal & III & $\begin{array}{l}\text { Ocasionalmente anemia } \\
\text { hemolítica aguda* }\end{array}$ & Moderada/deficiente & $\begin{array}{l}\text { Muito frequente } \\
\text { em áreas de alta } \\
\text { prevalência da Malária }\end{array}$ & $\begin{array}{l}\text { G6PD-Seattle, } \\
\text { G6PD-Canton, } \\
\text { G6PD-Rignano }\end{array}$ \\
\hline $60-150 \%$ do normal & IV & $\begin{array}{l}\text { Assintomática, anemia } \\
\text { aguda raramente } \\
\text { ocorre* }\end{array}$ & $\begin{array}{l}\text { Média a nenhuma/ } \\
\text { deficiente a normal }\end{array}$ & Frequência indefinida & $\begin{array}{l}\text { G6PD-Montalbano, } \\
\text { G6PD-Orissa }\end{array}$ \\
\hline$>150 \%$ do normal & $\mathrm{V}$ & Assintomática & $\begin{array}{l}\text { Nenhuma/Aumento da } \\
\text { atividade }\end{array}$ & Frequência indefinida & Não reportado \\
\hline \multicolumn{6}{|l|}{ Paciente } \\
\hline $\begin{array}{l}60-150 \% \text { do } \\
\text { normal** }\end{array}$ & IV & ----*** & $\begin{array}{l}\text { Média a nenhuma/ } \\
\text { deficiente a normal }\end{array}$ & ---- & ---- \\
\hline
\end{tabular}

Legenda: *dependente de fatores relacionados aoambiente, a alimentação e ao uso de medicamentos para induzir a crise hemolítica, Adaptado do Grupo de Trabalho da OMS (1989); **Com base nos achados clínicos, nos dados epidemiológicos, (distribuição geográfica) e na avaliação quantitativa da atividade residual da G6PD, com valor da atividade da G6PD de $84 \mathrm{mU} / 10^{9}$ eritrócitos (intervalo local de referência de 118 a 144, estes valores podem sofrer alterções para diferentes grupos populacionais, assim como eles são dependentes da metodologia adotada); ***Apresentados no relato do caso. Com base nas diretrizes da OMS, achados clínicos e mensuração quantitativa da atividade da G6PD nas hemácias são endossados para o diagnóstico de deficiência de G6PD ${ }^{8,13}$. 
A criança foi internada com história de febre por seis dias, associada a tosse seca, congestão brônquica (dificuldade em respirar), inapetência e vômitos. $\mathrm{O}$ exame físico revelou palidez $1+/ 4+$, desidratação $2+/ 4+$, febre $\left(38,8{ }^{\circ} \mathrm{C}\right)$, tendo a paciente apresentado-se prostrada e eupneica, com MV rude a ausculta pulmonar com crepitações em terço médio e ápice direito.

$\mathrm{Na}$ admissão, o hemograma completo da paciente mostrou anemia leve, anisocitose (RDW aumentado), e neutrofilia (dados mostrados como "Admissão" na Tabela 2). Outros resultados laboratoriais incluíram a Proteína $\mathrm{C}$ reativa (PCR) com resultado $>204 \mathrm{mg} / \mathrm{L}$ (valores de referência local $<9,0 \mathrm{mg} / \mathrm{L}$ ), íons $\mathrm{K}^{+}$inalterado, com $\mathrm{Na}^{+}$ de $136 \mathrm{mEq} / \mathrm{L}$ (valores de referência local de 136 a 145 $\mathrm{mEq} / \mathrm{L}$ ) e $\mathrm{Ca}^{+2}$ de $8,5 \mathrm{mg} / \mathrm{dl}$ (valores de referência local de 8,5 a $10,1 \mathrm{mg} / \mathrm{dL}$ ). Foram observados valores normais de Alanina aminotransferase (ALT) e de lactato sérico e ligeira elevação nos níveis de Aspartato aminotransferase (AST: 44 U/L, valores de referência local de 15 a 37 U/L). Hemocultura negativa. Raio-X do tórax anormal, com ângulos costofrênicos livres, preservação da vasculatura pulmonar e consolidação do lobo superior direito. O diagnóstico final estabelecido foi de pneumonia lobar aguda.

Para o tratamento da pneumonia lobar aguda, Penicilina G Cristalina (Penicilina G potássica, 3600000 UI) foi administrada por via intravenosa (200 mg/kg/dia), com doses a cada 6 horas durante 10 dias. Após o tratamento da paciente, clinicamente foi observado a resolução da infecção pulmonar e ausência de anemia hemolítica aguda. O acompanhamento com hemograma mostrou restauração dos valores normais de neutrófilos, restauração aos níveis normais de hemoglobina e hematócrito (acompanhamento dos parâmetros hematológicos indicados como "4 dias depois" e "9 dias depois" na Tabela 2).

\section{DISCUSSÃO}

As infecções respiratórias agudas são as principais causas de morbidade e mortalidade na infância, principalmente em países de baixa e média rendas ${ }^{14}$. Dados da Organização Mundial de Saúde (OMS) mostram que nos últimos dez anos, cerca de um terço da mortalidade global em crianças (4 a 5 milhões de mortes anuais) foram devido à infecções respiratórias agudas ${ }^{3,8,15}$. A incidência de pneumonia em crianças que têm deficiência de G6PD exige uma avaliação mais detalhada quanto ao tratamento farmacológico adotado, uma vez que a terapia medicamentosa pode causar episódios de crise hemolítica ${ }^{2,8}$.

Como a primeira escolha de medicamento para o tratamento de pneumonia em crianças, recomenda-se o uso de penicilina $\mathrm{G}$ procaína intramuscular ou amoxicilina oral. Por outro lado, penicilina G Cristalina pode ser usada em crianças com mais de três anos de idade, devido à prevalência de Streptococcus pneumoniae como um possível agente etiológico de infecções respiratórias $\operatorname{agudas}^{16}$.

$\mathrm{Na}$ admissão da paciente, os valores alterados no hemograma podem ser devido a doença infecciosa instalada. O medicamento escolhido para o tratamento da paciente com pneumonia lobar aguda foi a penicilina $G$ cristalina. Após a instituição da terapia medicamentosa, houve sucesso do tratamento da pneumonia lobar aguda e não ocorrência de crise hemolítica ou hemólise tardia. Não foi observado o aumento de reticulócitos, que normalmente ocorre quando há crise hemolítica induzida pelo fármaco (24 a 48 horas após o início do tratamento) ${ }^{12}$.

A restrição quanto ao uso de alguns medicamentos em pacientes com deficiência de G6PD é um desafio para a instituição de um tratamento adequado na prática clínica. Sérias dificuldades são impostas à estes pacientes, devido a possível crise hemolítica que pode surgir a partir do estilo de vida, hábitos alimentares e uso de medicamentos. As doenças infecciosas são também um dos mais comuns fatores desencadeantes da crise hemolítica. Além disso, alguns medicamentos que são usados para tratá-las, também foram classificados ou ainda estão sendo investigados como possíveis agentes causadores de crise hemolítica ${ }^{11,12,17}$.

Atualmente, existem escassos dados documentados sobre a segurança ou risco do uso da penicilina em pacientes com deficiência de G6PD (Tabela 3) ${ }^{18,19}$. A antibioticoterapia, parte de protocolos perioperatórios, tem levantado a questão do uso de penicilina por estes pacientes. Um estudo tem mostrado que, em doses terapêuticas e/ou para pacientes que são classificados como classes IV e V para deficiência de G6PD, a penicilina pode ser segura ${ }^{18}$. Por outro lado, outro estudo tem demonstrado que a penicilina não é segura ${ }^{19}$.

$\mathrm{O}$ risco de crise hemolítica em pacientes com deficiência de G6PD depende das características do fármaco, bem como da classe de variante da enzimopatia (niveis de atividade resídual da enzima). Alguns fármacos, por induzir a depleção dos niveis de GSH, podem comprometer um dos mais importantes sistemas antioxidantes em humanos. Esta depleção sistêmica de GSH pode ser somada a depleção intra-eritrocitária, por causa da dificiência de G6PD ${ }^{11,12}$. Ainda, além de em altas doses a penicilina ser conhecida por poder induzir um quadro de hemólise via resposta imune (envolvendo a formação de anticorpos e a participação do sistema complemento, portanto, diferindo da crise hemolítica induzida pela administração de penicilina em pacientes com deficiência de G6PD), um considerável efeito inibitório direto deste antibiótico sobre a atividade da enzima G6PD $\left(\mathrm{IC}_{50}=17,71 \mathrm{~m} M\right)$ tem sido reportado ${ }^{20}$.

Muitas variantes de G6PD foram descritas com um abrangente intervalo nos níveis de atividade da enzima e consequente diferentes quadros clínicos associadas. Além disso, os níveis da atividade enzimática pode variar 
Nogueira CF, et al. Uso da penicilina para tratar uma pneumonia lobar em paciente pediátrico com deficiência .

devido ao fenómeno de inativação do cromossomo X (mosaicismo $)^{10}$. No momento da utilização da penicilina, a restrição imposta pelos níveis de atividade de G6PD pode ser crucial para a sobrevivência das hemácias, uma vez que o grau do estresse oxidativo pode gerar diferentes quadros de crise hemolítica ${ }^{11-13,18,19}$.

Tabela 2. Parâmetros hematológicos seriais da paciente após a instituição de uma terapia sequencial de 10 dias com a penicilina

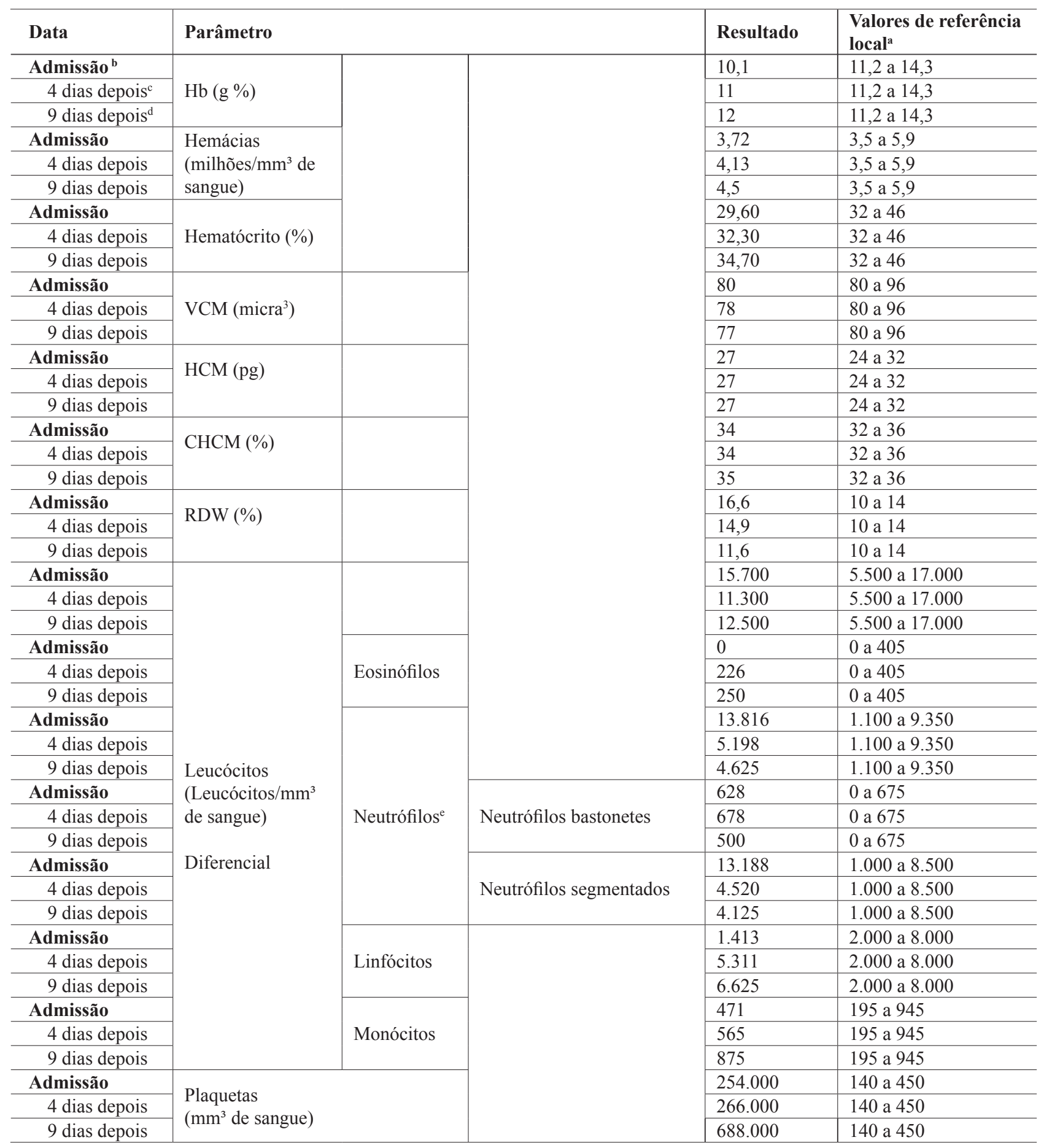

Legenda: ${ }^{a}$ Valores pediátricos de referência local com base na idade da paciente; ${ }^{\mathrm{b} S o b}$ admissão ${ }^{\mathrm{c}} \mathrm{e}$ dApós instituição de uma terapia sequencial com

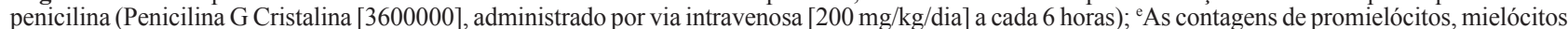
e metamielócitos foram de $0 / \mathrm{mm}^{3}$ para todos os três exames (intervalo de referência $\left.=0 / \mathrm{mm}^{3}\right) ; \mathrm{Hb}=$ hemoglobina; VCM = volume corpuscular médio; HCM = hemoglobina corpuscular média; CHCM = concentração de hemoglobina corpuscular média; RDW = Índice de anisocitose. Na admissão, os parâmetros hematológicos mostraram leve anemia, anisocitose, e neutrofilia. Até nove dias após a instituição de uma terapia sequencial de 10 dias com penicilina (3 e 8 dias mais tarde) os parâmetros hematológicos mostraram restauração dos valores normais de neutrófilos, a restauração de níveis normais de hemoglobina e hematócrito. 
Tabela 3. Estratégias de pesquisa conduzida para a revisão sistemática sobre o tema "uso de penicilina em pacientes com deficiência de glicose-6-fosfato desidrogenase (G6PD)" performada em diferentes bases de dados/domínio público

\begin{tabular}{|c|c|c|c|c|c|c|}
\hline Banco de dado & Palavras-chave & $\begin{array}{l}\text { Resultados } \\
\text { retornados }\end{array}$ & Período & $\begin{array}{l}\text { Critérios de } \\
\text { inclusão }\end{array}$ & $\begin{array}{l}\text { Critérios de } \\
\text { exclusão }\end{array}$ & Achados relevantes \\
\hline $\begin{array}{l}\text { PubMed } \\
\text { (via PubMed) }\end{array}$ & $\begin{array}{l}\text { (Penicillin) AND (glucose-6- } \\
\text { phosphate dehydrogenase (G6PD) } \\
\text { deficiency) }\end{array}$ & $\begin{array}{l}10 \\
\operatorname{artigos} *\end{array}$ & $\begin{array}{l}\text { Sem } \\
\text { restrição }\end{array}$ & $\begin{array}{l}\text { Pertinência/ } \\
\text { relevância } \\
\text { ao tema }\end{array}$ & $\begin{array}{l}\text { Texto } \\
\text { integral não } \\
\text { disponível }\end{array}$ & $\begin{array}{l}\text { Nada reportando sobre } \\
\text { o uso da penicilina } \\
\text { em pacientes com } \\
\text { deficiência de G6PD }\end{array}$ \\
\hline $\begin{array}{l}\text { Embase } \\
\text { (via OVID) }\end{array}$ & $\begin{array}{l}\text { 'Penicillin'/exp or 'Penicillin' } \\
\text { and ('glucose-6-phosphate } \\
\text { dehydrogenase (G6PD) deficiency'/ } \\
\text { exp or 'glucose-6-phosphate } \\
\text { dehydrogenase (G6PD) deficiency') }\end{array}$ & $\begin{array}{l}0 \\
\text { artigos }\end{array}$ & $\begin{array}{l}\text { Sem } \\
\text { restrição }\end{array}$ & $\begin{array}{l}\text { Pertinência/ } \\
\text { relevância } \\
\text { ao tema }\end{array}$ & $\begin{array}{l}\text { Texto } \\
\text { integral não } \\
\text { disponível }\end{array}$ & $\begin{array}{l}\text { Sem achados/ Não } \\
\text { conclusivos }\end{array}$ \\
\hline $\begin{array}{l}\text { Lilacs } \\
\text { (via Bireme) }\end{array}$ & $\begin{array}{l}\text { (tw:(Penicillin)) AND (tw:( glucose- } \\
\text { 6-phosphate dehydrogenase (G6PD) } \\
\text { deficiency)) }\end{array}$ & $\begin{array}{l}3 \\
\text { artigos }\end{array}$ & $\begin{array}{l}\text { Sem } \\
\text { restrição }\end{array}$ & $\begin{array}{l}\text { Pertinência/ } \\
\text { relevância } \\
\text { ao tema }\end{array}$ & $\begin{array}{l}\text { Texto } \\
\text { integral não } \\
\text { disponível }\end{array}$ & $\begin{array}{l}\text { Nada reportando sobre } \\
\text { o uso da penicilina } \\
\text { em pacientes com } \\
\text { deficiência de G6PD }\end{array}$ \\
\hline $\begin{array}{l}\text { Cochrane } \\
\text { Library }\end{array}$ & $\begin{array}{l}\text { (Penicillin) AND (glucose-6- } \\
\text { phosphate dehydrogenase (G6PD) } \\
\text { deficiency) }\end{array}$ & $\begin{array}{l}1 \\
\text { artigo }\end{array}$ & $\begin{array}{l}\text { Sem } \\
\text { restrição }\end{array}$ & $\begin{array}{l}\text { Pertinência/ } \\
\text { relevância } \\
\text { ao tema }\end{array}$ & $\begin{array}{l}\text { Texto } \\
\text { integral não } \\
\text { disponível }\end{array}$ & $\begin{array}{l}\text { Nada reportando sobre } \\
\text { o uso da penicilina } \\
\text { em pacientes com } \\
\text { deficiência de G6PD }\end{array}$ \\
\hline $\begin{array}{l}\text { Web of } \\
\text { Science }\end{array}$ & $\begin{array}{l}\text { (Penicillin) AND (glucose-6- } \\
\text { phosphate dehydrogenase (G6PD) } \\
\text { deficiency) }\end{array}$ & $\begin{array}{l}0 \\
\text { artigos }\end{array}$ & $\begin{array}{l}\text { Sem } \\
\text { restrição }\end{array}$ & $\begin{array}{l}\text { Pertinência/ } \\
\text { relevância } \\
\text { ao tema }\end{array}$ & $\begin{array}{l}\text { Texto } \\
\text { integral não } \\
\text { disponível }\end{array}$ & $\begin{array}{l}\text { Sem achados/ Não } \\
\text { conclusivos }\end{array}$ \\
\hline $\begin{array}{l}\text { Science } \\
\text { Direct }\end{array}$ & $\begin{array}{l}\text { (Penicillin) AND (glucose-6- } \\
\text { phosphate dehydrogenase (G6PD) } \\
\text { deficiency) }\end{array}$ & $\begin{array}{l}319 \\
\text { artigos }\end{array}$ & $\begin{array}{l}\text { Sem } \\
\text { restrição }\end{array}$ & $\begin{array}{l}\text { Pertinência/ } \\
\text { relevância } \\
\text { ao tema }\end{array}$ & $\begin{array}{l}\text { Texto } \\
\text { integral não } \\
\text { disponível }\end{array}$ & $\begin{array}{l}\text { Apenas } 1 \text { artigo } \\
\text { abordando o tema: } \\
\text {-Penicilina segura }^{18 * *}\end{array}$ \\
\hline OMIM & $\begin{array}{l}\text { (Penicillin) AND (glucose-6- } \\
\text { phosphate dehydrogenase (G6PD) } \\
\text { deficiency) }\end{array}$ & $\begin{array}{l}0 \\
\text { artigos }\end{array}$ & $\begin{array}{l}\text { Sem } \\
\text { restrição }\end{array}$ & $\begin{array}{l}\text { Pertinência/ } \\
\text { relevância } \\
\text { ao tema }\end{array}$ & $\begin{array}{l}\text { Texto } \\
\text { integral não } \\
\text { disponível }\end{array}$ & $\begin{array}{l}\text { Sem achados/ Não } \\
\text { conclusivos }\end{array}$ \\
\hline $\begin{array}{l}\text { SciELO } \\
\text { (via SciELO) }\end{array}$ & $\begin{array}{l}\text { (Penicillin) AND (glucose-6- } \\
\text { phosphate dehydrogenase (G6PD) } \\
\text { deficiency) }\end{array}$ & $\begin{array}{l}0 \\
\text { artigos }\end{array}$ & $\begin{array}{l}\text { Sem } \\
\text { restrição }\end{array}$ & $\begin{array}{l}\text { Pertinência/ } \\
\text { relevância } \\
\text { ao tema }\end{array}$ & $\begin{array}{l}\text { Texto } \\
\text { integral não } \\
\text { disponível }\end{array}$ & $\begin{array}{l}\text { Sem achados/ Não } \\
\text { conclusivos }\end{array}$ \\
\hline CINAHL & $\begin{array}{l}\text { (Penicillin) AND (glucose-6- } \\
\text { phosphate dehydrogenase (G6PD) } \\
\text { deficiency) }\end{array}$ & $\begin{array}{l}0 \\
\text { artigos }\end{array}$ & $\begin{array}{l}\text { Sem } \\
\text { restrição }\end{array}$ & $\begin{array}{l}\text { Pertinência/ } \\
\text { relevância } \\
\text { ao tema }\end{array}$ & $\begin{array}{l}\text { Texto } \\
\text { integral não } \\
\text { disponível }\end{array}$ & $\begin{array}{l}\text { Sem achados/ Não } \\
\text { conclusivos }\end{array}$ \\
\hline $\begin{array}{l}\text { MEDLINE } \\
\text { (via Bireme) }\end{array}$ & $\begin{array}{l}\text { (Penicillin) AND (glucose-6- } \\
\text { phosphate dehydrogenase (G6PD) } \\
\text { deficiency) ou (tw:(Penicillin)) } \\
\text { AND (tw:( glucose-6-phosphate } \\
\text { dehydrogenase (G6PD) deficiency)) }\end{array}$ & $\begin{array}{l}0 \\
\text { artigos }\end{array}$ & $\begin{array}{l}\text { Sem } \\
\text { restrição }\end{array}$ & $\begin{array}{l}\text { Pertinência/ } \\
\text { relevância } \\
\text { ao tema }\end{array}$ & $\begin{array}{l}\text { Texto } \\
\text { integral não } \\
\text { disponível }\end{array}$ & $\begin{array}{l}\text { Sem achados/ Não } \\
\text { conclusivos }\end{array}$ \\
\hline Scopus & $\begin{array}{l}\text { (Penicillin) AND (glucose-6- } \\
\text { phosphate dehydrogenase (G6PD) } \\
\text { deficiency) }\end{array}$ & $\begin{array}{l}0 \\
\text { artigos }\end{array}$ & $\begin{array}{l}\text { Sem } \\
\text { restrição }\end{array}$ & $\begin{array}{l}\text { Pertinência/ } \\
\text { relevância } \\
\text { ao tema }\end{array}$ & $\begin{array}{l}\text { Texto } \\
\text { integral não } \\
\text { disponível }\end{array}$ & $\begin{array}{l}\text { Sem achados/ Não } \\
\text { conclusivos }\end{array}$ \\
\hline BIOSIS & $\begin{array}{l}\text { (Penicillin) AND (glucose-6- } \\
\text { phosphate dehydrogenase (G6PD) } \\
\text { deficiency) }\end{array}$ & $\begin{array}{l}0 \\
\text { artigos }\end{array}$ & $\begin{array}{l}\text { Sem } \\
\text { restrição }\end{array}$ & $\begin{array}{l}\text { Pertinência/ } \\
\text { relevância } \\
\text { ao tema }\end{array}$ & $\begin{array}{l}\text { Texto } \\
\text { integral não } \\
\text { disponível }\end{array}$ & $\begin{array}{l}\text { Sem achados/ Não } \\
\text { conclusivos }\end{array}$ \\
\hline $\mathrm{HNO}$ & $\begin{array}{l}\text { (Penicillin) AND (glucose-6-phos- } \\
\text { phate dehydrogenase (G6PD) } \\
\text { deficiency) }\end{array}$ & $\begin{array}{l}14 \\
\text { artigos }\end{array}$ & $\begin{array}{l}\text { Sem } \\
\text { restrição }\end{array}$ & $\begin{array}{l}\text { Pertinência/ } \\
\text { relevância } \\
\text { ao tema }\end{array}$ & $\begin{array}{l}\text { Texto } \\
\text { integral não } \\
\text { disponível }\end{array}$ & $\begin{array}{l}\text { Apenas } 1 \text { artigo } \\
\text { abordando o tema: } \\
\text {-Penicilina não } \\
\text { segura }^{19 * *}\end{array}$ \\
\hline
\end{tabular}

Legenda: Última pesquisa no PubMed em 2 de outubro de 2015; Última pesquisa no Embase em 2 de outubro de 2015; Última pesquisa no Lilacs em 2 de outubro de 2015; Última pesquisa no Cochrane Library em 2 de outubro de 2015; Última pesquisa no Web of Science em 2 de Outubro de 2015; Última pesquisa no Science Direct em 2 de outubro de 2015; Última pesquisa no OMIM em 2 de outubro de 2015; Última pesquisa no SciELO em 3 de outubro de 2015; Última pesquisa no CINAHL em 3 de outubro de 2015; Última pesquisa no MEDLINE em 3 de outubro de 2015; Última pesquisa no Scopus em 3 de outubro de 2015; Última pesquisa no BIOSIS em 3 de outubro de 2015; Última pesquisa no HNO em 3 de outubro de 2015 ; **Nestes casos, existem outras co-morbidades/associação de tratamentos farmacológicos que devem ser considerados. *Apenas artigos publicados foram aceitos como documentos. 


\section{CONCLUSÃO}

Neste estudo nós relatamos que, após o início da antibioticoterapia com penicilina, observou-se a resolução da pneumonia lobar aguda e não ocorrência de crise hemolítica na paciente com deficiência de G6PD, conduzindo a um bem-sucedido e seguro tratamento medicamentoso. Contudo, novos estudos podem contribuir para reforçar as evidências sobre uma possível segurança quanto ao uso da penicilina por parte de pacientes com deficiência de G6PD, desde que a literatura pertinente ainda não traz uma ampla discussão sobre o tema e dados mais conclusivos para cada caso em particular. $\mathrm{O}$ prognóstico destes pacientes estão diretamente relacionados ao diagnóstico precoce desta enzimopatia, bem como das comorbidades ou de outras condições crônicas associadas ao longo da vida, permitindo assim a prevenção de episódios hemolíticos, através da escolha de uma farmacoterapia adequada e segura.

Financiamento: O estudo não recebeu financiamento.

Conflitos de interesse: Os autores declaram não haver conflitos de interesse.

Contribuição dos autores: Nogueira CFN, Aureliano APMS, LMTL. Acompanhamento do caso/obtenção dos dados; Cerdeira CD. Redação do manuscrito, análise e interpretação dos dados e revisão crítica do manuscrito; Silvério ACP, Santos GB. Orientação do trabalho/discentes.

\section{REFERÊNCIAS}

1. Al-Abdi SY, Alsaigh AS, Aldawoud FL, Sadiq AAA. Lower reference limits of quantitative Cord glucose-6-phosphate dehydrogenase estimated from healthy term neonates according to the clinical and laboratory standards institute guidelines: a cross sectional retrospective study. BMC Pediatrics. 2013;13:137. doi: 10.1186/1471-2431-13-137.

2. Alving AS, Carson PE, Flanagan CL, Ickes CE. Enzymatic deficiency in primaquine-sensitive erythrocytes. Science. 1956;124:484-5.

3. Beutler E. Glucose-6-phosphate dehydrogenase deficiency: a historical perspective. Blood. 2008;111:16-24. doi: 10.1182/ blood-2007-04-077412.

4. Peng Q, Li S, Ma K, Li W, Ma Q, et al. Large Cohort Screening of G6PD deficiency and the mutational spectrum in the Dongguan District in Southern China. PLoS ONE. 2015;10:e0120683. doi: 10.1371/journal.pone.0120683.

5. Luzzatto L, Metha A, Vulliamy T. Glucose 6-phosphate dehydrogenase deficiency. In: Scriver CR, Beaud, Sly WS, et al., editors. The metabolic and molecular bases of inherited disease. 8th ed. Columbus: McGraw-Hill; 2001. p.4517-53.

6. Cheng M, Ho HY, Liu HY, Chiu DTY, Lin HY. Impaired neutrophil extracellular traps formation of neutrophils from G6PD deficient patients. Free Radic Biol Med. 2010;49:1-2. doi: 10.1016/j.freeradbiomed.2010.10.405.

7. Cheng ML, Ho HY, Lin HY, Lai YC, Chiu DT. Effective NET formation in neutrophils from individuals with G6PD Taiwan-Hakka is associated with enhanced NADP $(+)$ biosynthesis. Free Radic Res. 2013;47:699-709. doi: 10.3109/10715762.2013.816420.

8. WHO Working Group. Glucose-6-phosphate dehydrogenase deficiency. Bull World Health Organ. 1989;67:601-11. Available from: https://www.ncbi.nlm.nih.gov/pmc/articles/ PMC2491315/pdf/bullwho00064-0002.pdf.

9. Wolach B, Ashkenazi M, Grossmann R, Gavrieli R, Friedman Z, Bashan N, Roos D. Diurnal fluctuation of leukocyte G6PD Activity. A possible explanation for the normal neutrophil bactericidal activity and the low incidence of pyogenic infections in patients with severe G6PD deficiency in Israel. Pediatr Res. 2004;55:807-13. doi: 10.1203/01. PDR.0000120680.47846.47
10.Peters AL, Van Noorden CJF. Glucose-6-phosphate dehydrogenase deficiency and malaria: cytochemical detection of heterozygous G6PD deficiency in women. J Histochem Cytochem. 2009;57:1003-11. doi: 10.1369/jhc.2009.953828.

11. Youngster I, Arcavi L, Schechmaster R, Akayzen Y, Popliski H, Shimonov J, Beig S, Berkovitch M. Medications and glucose6-phosphate dehydrogenase deficiency: an evidence-based review. Drug Saf. 2010;33:713-26. doi: 10.2165/11536520000000000-00000.

12. Elyassi AR, Rowshan HH. Perioperative management of the glucose-6-phosphate dehydrogenase deficient patient: a review of literature. Anesth Prog. 2009;56:86-91. doi: 10.2344/00033006-56.3.86.

13. Kafkas NV, Liakos CI, Mouzarou AG. Antiplatelet and invasive treatment in patients with glucose-6-phosphate dehydrogenase (G6PD) deficiency and acute coronary syndrome. The safety of Aspirin. J Clin Pharm Ther. 2015;40:349-52. doi: 10.1111/ jcpt.12262.

14. Shann F. Etiology of severe pneumonia in children in developing countries. Pediatr Infect Dis. 1986;5:247-52.

15. Berman S, Shanks MB, Feiten D, Horgan JG, Rumack C. Acute respiratory during the first three months of life: clinical radiologic and physiologic predictors of etiology. Pediatr Emerg Care. 1990;6:179-82.

16. Diretrizes brasileiras em pneumonia adquirida na comunidade em pediatria - 2007. J Bras Pneumol. 2007;33(Suppl 1):31-50. doi: 10.1590/S1806-37132007000700002.

17. Cappellini MD, Fiorelli G. Glicose-6-fosfato desidrogenase. Lancet. 2008;371(9606):64-74. doi: 10.1016/S01406736(08)60073-2.

18. Martin LD, Casella ES. Anesthesia and glucose-6-phosphate dehydrogenase deficiency in a child with congenital heart disease. J Cardiothorac Vasc Anesth. 1991;5:596-9.

19.Depta AL, Erdös G, Werner C. Anästhesie bei patienten mit glukose-6-phosphat-dehydrogenase-mangel: fallbericht und perioperatives anästhesiologisches management. Anaesthesist. 2006;55:550-4. doi: 10.1007/s00101-005-0971-6.

20. Çiftçi M, Türkoğlu V, Aldemir S. Effects of some antibiotics on glucose 6-phosphate dehydrogenase in sheep liver. Vet Med Czech. 2002;47(10-11):283-8.

Artigo recebido em: 15.03.2016

Artigo aceito em: 17.05.2017 